\title{
Dynamic assembly of class II hydrophobins from T.reesei at the air-water interface
}

${ }^{17}$ Hendrik Hähl, ${ }^{1,2}$ Alessandra Griffo, ${ }^{3}$ Neda Safaridehkohneh, ${ }^{1}$ Jonas Heppe, ${ }^{1,4}$ Sebastian Backes, ${ }^{5}$ Michael Lienemann, ${ }^{2}$ Markus B. Linder, ${ }^{3}$ Ludger Santen, ${ }^{2,6+t}$ Päivi Laaksonen* and ${ }^{1}$ Karin Jacobs

${ }^{1}$ Department of Experimental Physics, Saarland University, 66041 Saarbrücken, Germany, ${ }^{2}$ Department of Bioproducts and Biosystems, Aalto University, PO Box 16100, FI-00076 AALTO, Finland

${ }^{3}$ Department of Theoretical Physics, Saarland University, 66041 Saarbrücken, Germany, ${ }^{4}$ Federal Institute for Material Research and Testing (BAM), Unter den Eichen 87, 12205 Berlin, Germany

${ }^{5}$ VTT Technical Research Centre of Finland Ltd., Espoo 02150, Finland

${ }^{6}$ HAMK Tech, Häme University of Applied Sciences, Po Box 230, Hämeenlinna, 13101, Finland
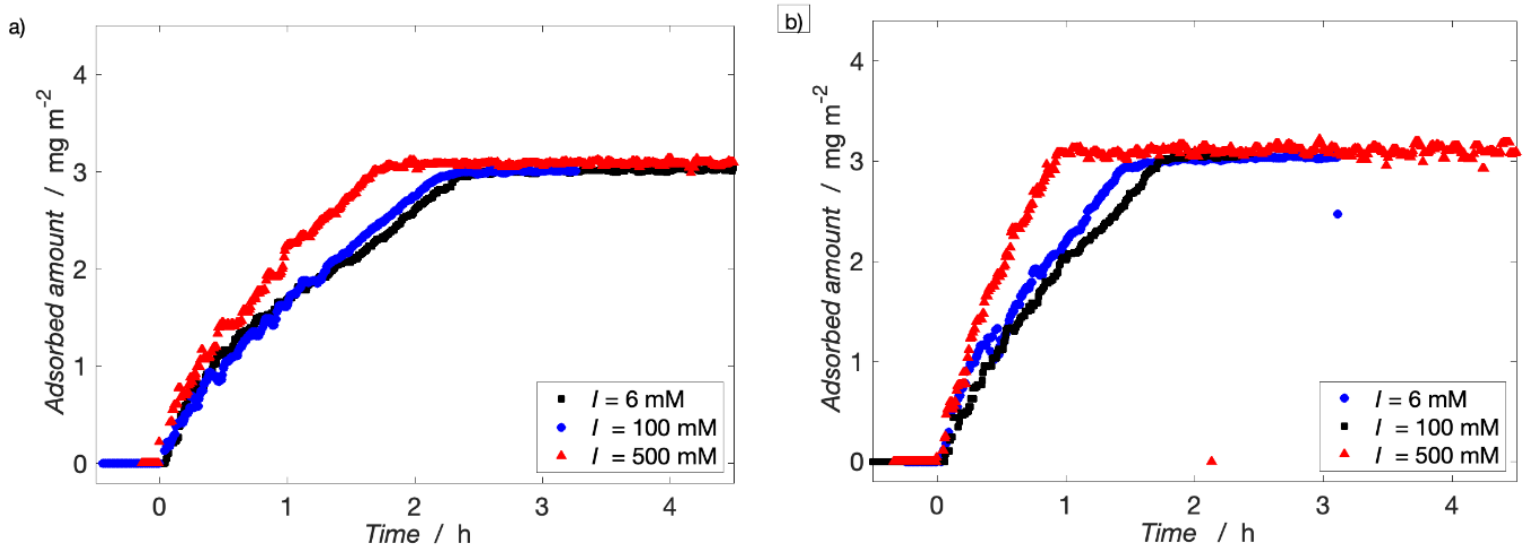

Figure S1. Adsorption kinetics of a) WT HFBI and b) HFBI-D30N-K32Q at protein solution concentration of $0.2 \mu \mathrm{M}$ and varied ionic strength (black: $6 \mathrm{mM}$, blue: $100 \mathrm{mM}$ and red: $500 \mathrm{mM}$ ) measured via time resolved ellipsometry measurement. 


\section{Surface pressure}
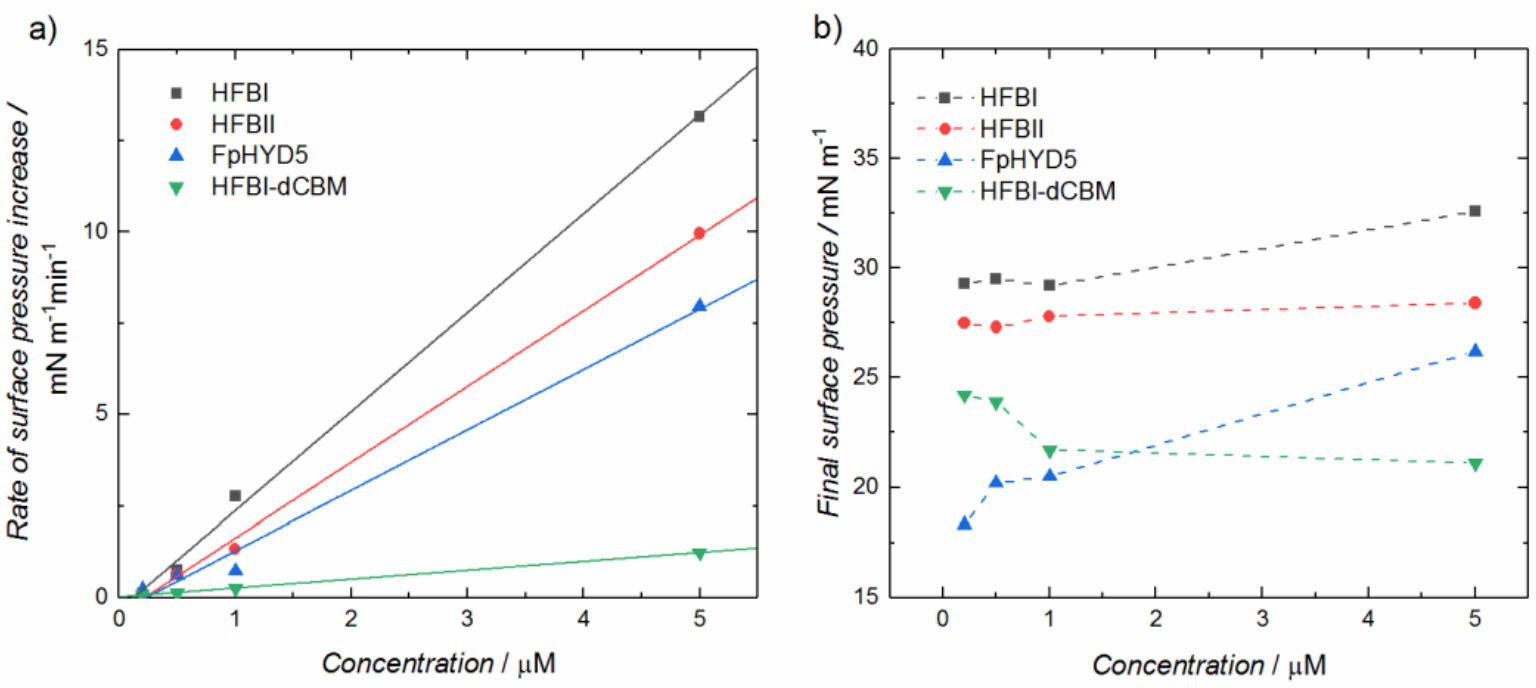

Figure S2.a) The rate of surface pressure increase for HFBI, HFBII, FpHYD5 and HFBIdCBM. The lines are linear fits to the data points. b) Saturation value of the surface pressure for HFBI, HFBII, FpHYD5 and HFBI-dCBM. The dashed lines are guides to the eye.

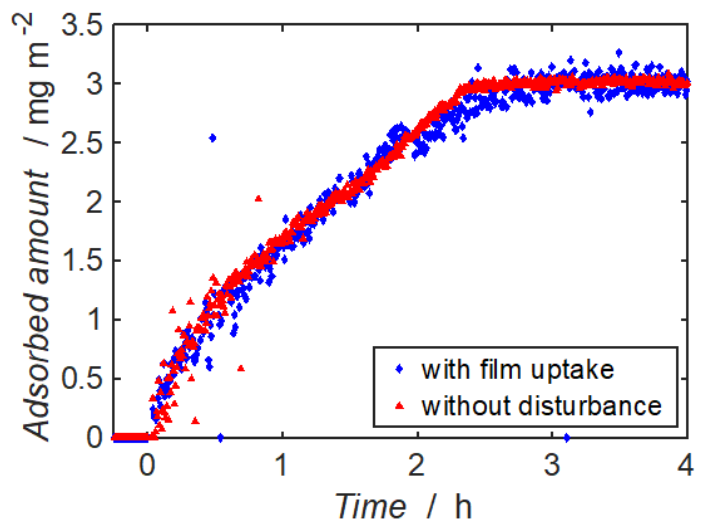

Figure S3. Comparison of ellipsometry measurements of HFBI (c $=0.2 \mu \mathrm{M})$ adsorbing to the air-water interface while picking up the Langmuir-Schaefer films for AFM (blue diamonds) and without disturbance (red triangles, see also Fig. 1 b). 
a)

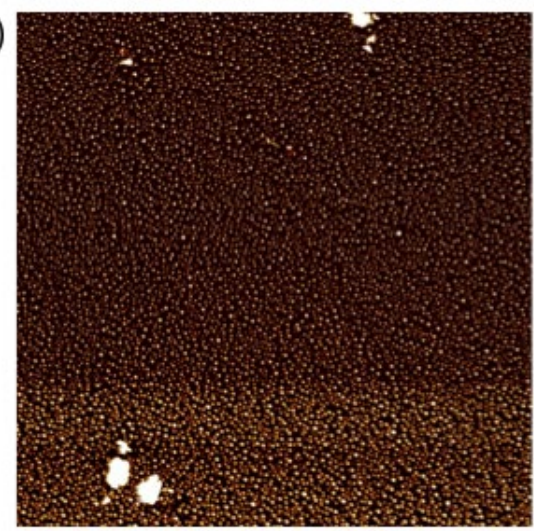

b)

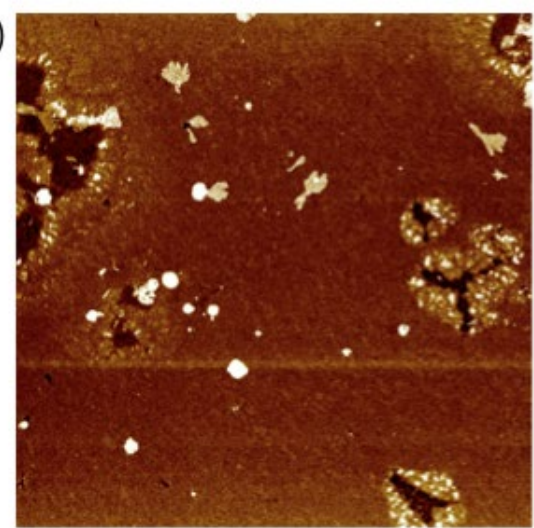

$10.0 \mathrm{~nm}$

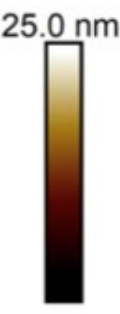

Figure S4. AFM images (size: $10 \mu \mathrm{m} \times 10 \mu \mathrm{m}$ ) of Langmuir-Schaefer films of HFBI-dCBM a) ca. $15 \mathrm{~min}$. and b) $10 \mathrm{~h}$ after start of the adsorption measurements. The films were prepared as the HFBI films presented in Fig. 5. In contrast to the HFBI films, no cluster growth can be observed. In a) agglomerations of proteins can be seen, very likely formed during the drying process.

a)

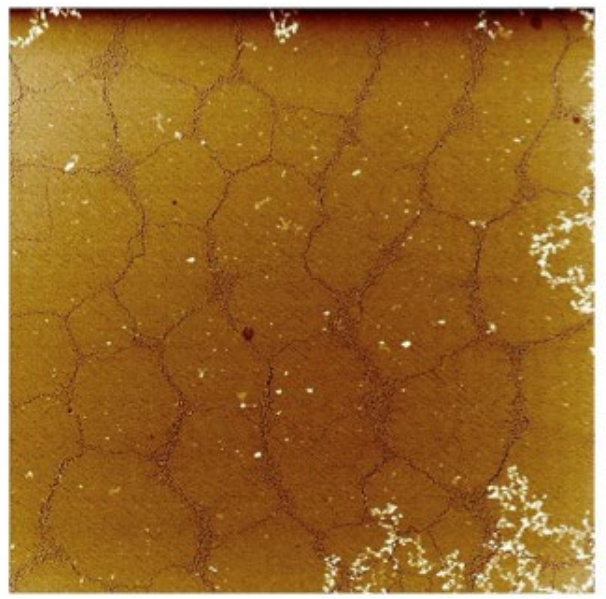

b)

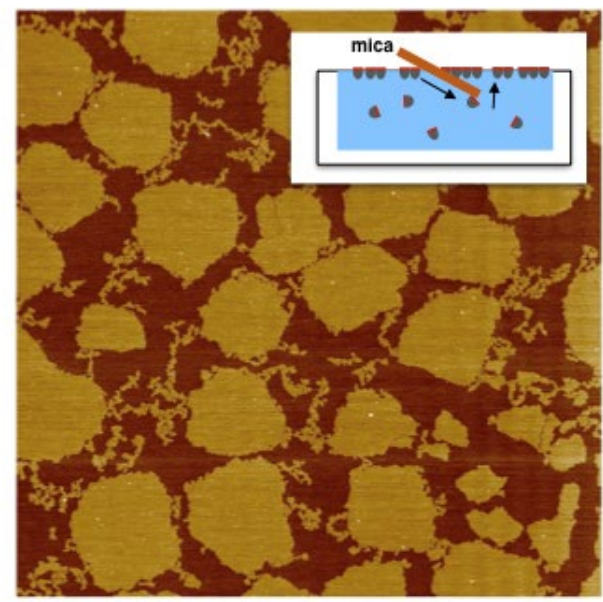

Figure S5. AFM images (size: $10 \mu \mathrm{m} \times 10 \mu \mathrm{m}$ ) of Langmuir-Schaefer films of HFBI more than $22 \mathrm{~h}$ after protein injection into the solution $(c=0.2 \mu \mathrm{M})$. a) The film was prepared as the films shown in Fig. 5 in the main manuscript. A completely saturated film is observed still showing boundaries between individual clusters. b) This film was prepared on a freshly cleaved mica sheet which was first introduced in the solution and then lifted in order to bring the hydrophilic parts of the hydrophobins in contact with the mica (see inset sketch). Due to this procedure, the interface is heavily disturbed and clusters are separated. In comparison to the images in Fig. 5, the hydrophobic and hydrophilic regions are inverted: Here, the cluster surfaces are hydrophobic, whereas the substrate around the clusters is hydrophilic. The appearance of clusters in both a) and $b$ ) shows that the clusters are not induced by the moving three-phase-contact line of water residuals after film uptake but form spontaneously at the air-water interface. 


\section{Hamiltonian details}

In our model, each protein interacts with the other proteins on the same layer (interaction between proteins on the interface and the subsurface will be included in a future publications). We consider both isotropic and anisotropic kind of interactions. The isotropic part of interactions is described in the framework of the Derjaguin-Landau-Verwey-Overbeek (DLVO) theory $[1,2]$. Which includes van der Waals, steric repulsion, and Coulomb interactions. The structure of the potential is given by:

$$
\begin{gathered}
U(r)=U_{v d w}+U_{\text {coulomb }}+U_{\text {steric }} \\
U_{v d w}=\frac{-B \times R}{12 \times(r-2 R)} \\
U_{\text {Coulomb }}=\frac{A_{e l}\left(L_{D}\right) R^{2}}{r} e^{\frac{-(r-2 R)}{L_{D}}} \\
U_{\text {Steric }}=\frac{F}{(r-2 R)^{6}}
\end{gathered}
$$

Where $r$ is the center-to-center distance of two interacting particles. The cutoff-radius of the calculations is chosen such that a further increase of the distance does not change the total energy quantitatively.

The anisotropic part of interactions between two adsorbed proteins has been introduced into the Hamiltonian by considering a spin-type model. For each protein, six discrete in-plane orientations are possible. Two adjacent proteins that have the same orientation attract each other. For two proteins, with orientations $S_{1}$ and $S_{2}$, the spin contribution is given by:

$$
U_{\text {Spin }}=J_{0} \delta_{S_{1} S_{2}}
$$

The relative value of $J_{0}$ is the adjusted according to the isotropic part of energy. $\delta_{S_{1} S_{2}}$ denotes the Kronecker delta function. A similar spin model has been used in Magakar et al. [3].

\section{REFERENCES}

(1) Bellion, M.; Santen, L.; Mantz, H.; Hähl, H.; Quinn, A.; Nagel, A.; Gilow, C.; Weitenberg, C.; Schmitt, Y.; Jacobs, K. Protein adsorption on tailored substrates: long-range forces and conformational changes. J. Phys: Condens. Matter. 2008, 20 404226, 1-11.

(2) Zbigiew, A.; Weronsski, P. Application of the DLVO theory for particle deposition problems. Advances in Colloid and Interfaces Science. 1999, 83, 137-226.

(3) Magarkar, A.; Mele, N.; Abdel-Rahman, N.; Butcher, S.; Torkkeli, M.; Serimaa, R.; Paananen, A.; Linder, M.; Bunker, A. Hydrophobin Film Structure for HFBI and HFBII and Mechanism for Accelerated Film Formation. PLOS Comput. Biol. 2014, 10 (7), e1003745. 